\title{
What the lattice can tell us about nucleon structure
}

\author{
W. Schroers ${ }^{a}$ \\ a John von Neumann-Institut für Computing NIC/DESY, 15738 Zeuthen, Germany
}

This review focuses on the current status of lattice calculations of three observables which are both phenomenologically and experimentally relevant and have been scrutinized recently. These three observables are the nucleon electromagnetic form factors, the momentum fraction, $\langle x\rangle_{\mathrm{u}-\mathrm{d}}$, and the nucleon axial coupling, $g_{A}$.

DESY preprint: DESY 05-243

\section{INTRODUCTION}

Understanding nucleon structure using lattice simulations has progressed tremendously over the past years. Both the availability of new machines and the improvement of algorithms and techniques have contributed to the progress. The major challenge of contemporary lattice simulations remains to perform simulations at sufficiently small quark masses. For example, the discovery of Ginsparg-Wilson fermions has made it possible to calculate at quark masses which are substantially lighter than what used to be accessible before.

On the other hand, chiral extrapolation techniques are increasingly better understood. The current status of research depends on the quantity under consideration. For a recent review on chiral extrapolation techniques for nucleon structure, see [1]. For a general review on lattice chiral perturbation techniques, see [2]. An interesting new development is also the application of models, like the chi- ral quark-soliton model in 3]. However, such models have not yet been used to make contact with nucleon structure lattice data so far.

This presentation focuses on the current status of three observables characterizing the shape of the nucleon, the electromagnetic form factors, $F_{1}$ and $F_{2}$, the first moment of the nucleon spin-independent parton distribution, $\langle x\rangle_{\mathrm{u}-\mathrm{d}}$ and the axial coupling, $g_{A}$. The results for $\langle x\rangle_{\mathrm{u}-\mathrm{d}}$ will be quoted in the $\overline{\mathrm{MS}}$-scheme with a scale of $\mu=2 \mathrm{GeV}$. The discussion of the latter two quantities constitutes an update to the previous review [4].

For technical details of lattice methods see [567] and also 8] for a recent review. For calculations of GPDs going beyond experimentally accessible data, see references 9 10.

Results from different lattice studies are shown in table1. These studies combine a variety of different technologies and cover a wide range of parameters. While the early investigations were limited to linear chiral extrapolations from rather large 
Table 1

Compilation of different lattice investigations of $\langle x\rangle_{\mathrm{u}-\mathrm{d}}$ and $g_{A}$.

\begin{tabular}{lllll}
\hline Group \& Ref. & $m_{\pi}$ & Technique & $\langle x\rangle_{\mathrm{u}-\mathrm{d}}$ & $g_{A}$ \\
\hline Kentucky [1] & $?$ & Wilson (quenched) & - & $1.20(11)$ \\
KEK [12] & $>530 \mathrm{MeV}$ & Wilson (quenched) & - & $0.985(25)$ \\
QCDSF [5] & $>600 \mathrm{MeV}$ & Wilson (quenched) & $0.263(17)$ & $1.074(90)$ \\
LHPC [13] & $>650 \mathrm{MeV}$ & Wilson (full) & $0.269(23)$ & $1.031(81)$ \\
RBCK [14] & $>390 \mathrm{MeV}$ & DWF (quenched) & - & $1.212(27)$ \\
LHPC [15] & $>360 \mathrm{MeV}$ & Hybrid & $-{ }^{1}$ & $-^{1}$ \\
LHPC [16] & $>360 \mathrm{MeV}$ & Hybrid & - & $1.212(84)$ \\
QCDSF [6] & $>550 \mathrm{MeV}$ & CI-Wilson (quenched) & $0.245(19)$ & - \\
QCDSF [17] & $>550 \mathrm{MeV}$ & CI-Wilson (full) & - & $-{ }^{1}$ \\
QCDSF [18] & $>550 \mathrm{MeV}$ & CI-Wilson (full) & - & $-{ }^{2}$ \\
QCDSF [19] & $>300 \mathrm{MeV}$ & Overlap (quenched) & $-{ }^{1}$ & $-{ }^{1}$ \\
RBCK [20 21] & $>400 \mathrm{MeV}$ & DWF (quenched/full) & $-{ }^{3}$ & - \\
\hline Expen
\end{tabular}

Experimental values: $\langle x\rangle_{\mathrm{u}-\mathrm{d}}=0.154(3)[22], g_{A}=1.248(2)[23$, see also [24]

${ }^{1}$ Work in progress and/or no prediction quoted

${ }^{2}$ Constrained fit yields consistency with experiment

${ }^{3}$ Agreement found with experiment for $\langle x\rangle_{\mathrm{u}-\mathrm{d}} /\langle x\rangle_{\Delta \mathrm{u}-\Delta \mathrm{d}}$

quark masses, the latter studies either do not quote numbers from naive extrapolations or perform constrained fits using chiral perturbation theory.

\section{ELECTROMAGNETIC FORM FACTORS}

Understanding the behavior of the form factor ratio, $F_{2} / F_{1}$, has recently regained attention in the study of exclusive reactions. Arguments based on asymptotic scaling [25] result in scaling laws for $F_{1} \propto$ $Q^{-4}$ and $F_{2} \propto Q^{-6}$. Hence, one would expect $F_{2} / F_{1}$ to scale like $\propto Q^{-2}$. Recent experiments [26], however, have indicated that instead the data is more consistently described with a scaling relation like $F_{2} / F_{1} \propto Q^{-1}$. Using arguments from perturbative QCD, reference 27] finds an explicit expression for the scaling behavior of the form factor ratio which is consistent with the experimental data.

First lattice studies have confirmed this scaling behavior already at quark masses beyond $700 \mathrm{MeV}$ [10]. So far, the QCDSF group is currently investigating this ratio in full QCD using Clover fermions 28 29]. An obstacle is still provided by the fact that lattice data is only available for values of $Q^{2}$ smaller than $3 \mathrm{GeV}^{2}$. A preliminary feasibility study of the behavior of form factors at larger values of $Q^{2}$ has been performed in [15. It appears that the region of $Q^{2}>3 \mathrm{GeV}^{2}$ is currently not easily reached in lattice simulations.

Beyond this ratio, the computation of magnetic moments and rms-radii is pos- 
sible using lattice simulations today. Unlike the asymptotic ratio these quantities require data points at small values of $Q^{2}$ with sufficient accuracy. In principle, the quality of the lattice data is almost as good as for $g_{A}$ since the matrix elements do not involve any derivatives. However, at the moment there are only few sophisticated expressions from small quark mass expansions known. The direct comparison between lattice data with one variant of a small scale expansion at values of the pion mass beyond $550 \mathrm{MeV}$ has been presented in [30]. The lattice data in this publication, however, is quenched and it will be interesting to perform a careful check with unquenched data which will soon be available with good statistics in 29. For the case of magnetic moments, a quenched study in 31 has attempted a comparison between quenched and real QCD beyond the application of quenched chiral effective field theory. A different path to compute magnetic moments has been pursued in 32 .

It appears that still some technological improvements and an increased understanding of chiral techniques is necessary to finally arrive at quantitative predictions similar to those available for $g_{A}$.

\section{THE MOMENT $\langle x\rangle_{\mathrm{u}-\mathrm{d}}$}

As has been pointed out before in [4, almost all results for $\langle x\rangle_{\mathrm{u}-\mathrm{d}}$ systematically exceed the results quoted by phenomenologists by about $50 \%$. Given the variety of parameters and technologies used, neither finite size, nor unquenching or lattice artifacts are responsible for this discrepancy.
While an earlier study by the QCDSF collaboration using quenched Overlap fermions in 33] found a systematically smaller value than other publications, an update of this calculation in [19] finds results consistent with those of Wilson-type fermions. This discrepancy has been resolved in 34. It was found that the discrepancy can arise from considering a nonperturbative matching instead of leadingorder perturbative renormalization.

A mismatch similar to the one found in 33. has been reported by the LHPC collaboration in [15], see figure 3 in this reference. The data points for $\langle x\rangle_{\mathrm{u}-\mathrm{d}}$ lie on a straight line with no indication of any bending down. However, they are both inconsistent with the results at large quark masses and the experimental value. This paper also utilizes a leading order perturbative matching and it is possible that a non-perturbative calculation of the renormalization procedure will lead to a correction that makes the hybrid data consistent with the other calculations again.

It is interesting as the RBCK collaboration points out in 21] that the ratio $\langle x\rangle_{\mathrm{u}-\mathrm{d}} /\langle x\rangle_{\Delta \mathrm{u}-\Delta \mathrm{d}}$ is in complete agreement with the experimental value. This finding has also been reproduced by LHPC in 15. It could provide an indication that the problem affects both individual quantities in the same way by a common factor which cancels as the ratio is taken.

At this moment it is safe to conclude that no study so far has observed any evidence for a "bending down" of the momentum fraction as the quark mass is decreased. A possible scenario for such a 
behavior has been explored in 35. The mystery shrouding this quantity remains unsolved.

\section{THE AXIAL COUPLING $g_{A}$}

Unlike the observable discussed in the previous section, the understanding of the axial coupling has progressed much further. Although it is not yet possible to postdict the experimental value of $g_{A}$ from first principles alone, it has become feasible to connect expressions from chiral expansion techniques with lattice results.

The coupling $g_{A}$ is known to be very sensitive to finite-size effects which has first been observed in [14 and later confirmed by other groups in 17. and 36. Together with the dependence on the quark mass these finite size effects can accurately be modeled by chiral expansion techniques. These have now reached very sophisticated levels 173718.

The reference [16] reports a statistical residual error of $5 \%$ for a complete calculation of $g_{A}$ down to pion masses of $350 \mathrm{MeV}$ with a physical lattice size of $3.5 \mathrm{fm}$. This lattice size results in negligible finite-size effects. Performing a constrained threeparameter fit with an expression from chiral perturbation theory finally yields an error band of $7 \%$ at the physical value of the quark mass, cf. figure 2 in [16].

This analysis shows how lattice simulations can already today reproduce experimental values and gives rise to the hope that similar quantities will soon be equally well understood.

\section{CONCLUSIONS}

The dependence of the first moment of the spin-independent parton distribution $\langle x\rangle_{\mathrm{u}-\mathrm{d}}$ is still not understood and there is evidence that a "bending over" of the lattice data does not occur until very small values of the quark mass if ever. This situation remains a puzzle to both phenomenologists and lattice physicists.

However, lattice calculations are now reaching a turning point. For the first time, it is possible to present a convincing case for the matching of state-of-theart lattice data and sophisticated chiral expansion techniques. This has allowed to qualitatively and quantitatively understand the quark mass dependence of the nucleon axial coupling $g_{A}$.

A similar level can be expected to be reached for form factors in the region of small values of $Q^{2}$. This will allow for an accurate quantitative computation of rmsradii and magnetic moments. The scaling behavior of form factors at larger values of $Q^{2}$, however, appears to be a bigger obstacle and does not promise to be understood similarly well in the near future.

This presentation was supported in part by the DFG (Forschergruppe GitterHadronen-Phänomenologie and in part by the EU Integrated Infrastructure Initiative Hadron Physics (I3HP) under contract number RII3-CT-2004-506078. I thank the Alexander-von-Humboldt Foundation for their support and the Center for Theoretical Physics at MIT for their hospitality. 


\section{REFERENCES}

1. M. Göckeler, arXiv:hep-lat/0412013.

2. O. Bär, Nucl. Phys. Proc. Suppl. 140, 106 (2005).

3. K. Goeke, J. Ossmann, P. Schweitzer and A. Silva, arXiv:hep-lat/0505010.

4. W. Schroers, Nucl. Phys. A $\mathbf{7 5 5}$ (2005) 333.

5. M. Göckeler et al. [QCDSF], Phys. Rev. D 53 (1996) 2317.

6. M. Göckeler, R. Horsley, D. Pleiter, P.E.L. Rakow and G. Schierholz [QCDSF Collaboration], arXiv:hep-ph/0410187.

7. M. Göckeler et al. [QCDSF Collaboration], Nucl. Phys. A 755 (2005) 537.

8. R. Horsley, arXiv:hep-lat/0412007.

9. M. Göckeler et al. [QCDSF], Phys.

Rev. Lett. 92, 042002 (2004).

P. Hägler et al. [LHPC], Phys. Rev. D 68, 034505 (2003).

P. Hägler et al. [LHPC], Phys. Rev. Lett. 93, 112001 (2004).

J. W. Negele et al. [LHPC], Nucl. Phys. Proc. Suppl. 129, 910 (2004).

W. Schroers et al. [LHPC], Nucl. Phys. Proc. Suppl. 129, 907 (2004).

M. Göckeler et al. [QCDSF], Nucl. Phys. Proc. Suppl. 128, 203 (2004).

M. Göckeler et al. [QCDSF], Nucl. Phys. Proc. Suppl. 135, 156 (2004).

M. Göckeler et al. [QCDSF Collaboration], Nucl. Phys. Proc. Suppl. 140 (2005) 399.

P. Hägler, J. W. Negele, D. B. Renner, W. Schroers, T. Lippert and K. Schilling [LHPC Collaboration], Eur. Phys. J. A 24S1 (2005) 29.

M. Göckeler et al. [QCDSF Collabora- tion], Few Body Syst. 36 (2005) 111.

B. Bistrovic et al. [Lattice Hadron Physics Collaboration], J. Phys. Conf. Ser. 16 (2005) 150.

10. J. W. Negele et al. [LHPC], Nucl. Phys. Proc. Suppl. 128, 170 (2004).

11. K. F. Liu et al., Phys. Rev. D 49, 4755 (1994).

12. M. Fukugita, Y. Kuramashi, M. Okawa and A. Ukawa, Phys. Rev. Lett. 75 (1995) 2092.

13. D. Dolgov et al. [LHPC], Phys. Rev. D 66 (2002) 034506.

14. S. Sasaki, K. Orginos, S. Ohta and T. Blum [RBCK], Phys. Rev. D 68, 054509 (2003).

15. R. G. Edwards et al. [LHPC Collaboration], PoS LAT2005 (2005) 056.

16. R. G. Edwards et al. [LHPC Collaboration], arXiv:hep-lat/0510062.

17. A. A. Khan et al., Nucl. Phys. Proc. Suppl. 140, 408 (2005).

18. A. A. Khan et al., arXiv:hep-lat/0510061.

19. D. Galletly et al. [QCDSF Collaboration], arXiv:hep-lat/0510050.

20. S. Ohta and K. Orginos [RBCK], Nucl. Phys. Proc. Suppl. 140, 396 (2005).

S. Ohta and K. Orginos [RBCK], Nucl. Phys. Proc. Suppl. 129 (2004) 296.

21. K. Orginos, T. Blum and S. Ohta, arXiv:hep-lat/0505024

22. H. L. Lai et al., Phys. Rev. D 55, 1280 (1997).

M. Glück, E. Reya and A. Vogt, Eur. Phys. J. C 5, 461 (1998).

A. D. Martin, R. G. Roberts, 
W. J. Stirling and R. S. Thorne, Eur. Phys. J. C 23, 73 (2002).

23. J. Blümlein and H. Böttcher, Nucl. Phys. B 636 (2002) 225.

M. Glück, E. Reya, M. Stratmann and W. Vogelsang, Phys. Rev. D 63 (2001) 094005.

Y. Goto et al. [AAC], Phys. Rev. D 62, 034017 (2000).

M. Hirai, AIP Conf. Proc. 675, 365 (2003).

24. J. Blümlein, H. Böttcher and A. Guffanti, Nucl. Phys. Proc. Suppl. 135 (2004) 152.

25. S. J. Brodsky and G. R. Farrar, Phys. Rev. D 11 (1975) 1309.

G. P. Lepage and S. J. Brodsky, Phys. Rev. D 22 (1980) 2157.

26. O. Gayou et al. [Jefferson Lab Hall A Collaboration], Phys. Rev. Lett. 88 (2002) 092301.

O. Gayou et al., Phys. Rev. C 64 (2001) 038202.

27. A.V. Belitsky, X. d. Ji and F. Yuan, Phys. Rev. Lett. 91, 092003 (2003).

28. D. Pleiter, "Nucleon form factors from $n_{f}=2$ clover fermions", talk presented at the ILFT workshop at Jefferson Lab, Oct. 2005.

29. M. Göckeler et al., in preparation.

30. M. Göckeler, T.R. Hemmert, R. Horsley, D. Pleiter, P.E.L. Rakow, A. Schäfer and G. Schierholz [QCDSF Collaboration], Phys. Rev. D 71 (2005) 034508.

31. R.D. Young, D.B. Leinweber and A.W. Thomas, Phys. Rev. D 71 (2005) 014001.

32. F. X. Lee, R. Kelly, L. Zhou and
W. Wilcox, Phys. Lett. B 627, 71 (2005).

33. M. Gürtler et al., arXiv:hep-lat/0409164.

D. Galletly et al. [QCDSF-UKQCD], Nucl. Phys. Proc. Suppl. 129, 453 (2004).

34. M. Gürtler, R. Horsley, P. E. L. Rakow, C. J. Roberts, G. Schierholz and T. Streuer [QCDSF Collaboration], arXiv:hep-lat/0510045.

35. W. Detmold et al., Phys. Rev. Lett. 87, 172001 (2001).

36. D. B. Renner et al. [LHPC], Nucl. Phys. Proc. Suppl. 140, 255 (2005).

37. S.R. Beane and M.J. Savage, Phys. Rev. D 70 (2004) 074029.

W. Detmold and D.J.D. Lin, Phys. Rev. D 71 (2005) 054510. 